\title{
Estratégias de vigilância de um acidente envolvendo planta industrial de produção de ácido sulfúrico
}

\section{Surveillance strategies of an accident involving a sulfuric acid production plant}

\author{
Clarice Umbelino de Freitas ${ }^{1}$, Pedro José Villaça², Carolina Beltramini de Carvalho Donola³, \\ Débora Moraes Coelho ${ }^{3}$, Ana Claudia Furlan Mori ${ }^{3}$, Marlene Tursi Zampietro ${ }^{1}$, André Pereira Leite ${ }^{1}$
}

\begin{abstract}
Resumo
Após acidente ambiental em empresa produtora de ácido sulfúrico, realizou-se estudo transversal para investigação de sintomas respiratórios e irritativos na população. Aplicou-se questionário em amostra aleatória simples, em pessoas presentes no local no dia da ocorrência, no raio de $900 \mathrm{~m}$ do estabelecimento, considerando a direção dos ventos. Foi calculada a prevalência de sintomas segundo gradientes de $300 \mathrm{~m}$ e realizado teste de Chi-Quadrado para sintomas isolados e agregados. Os dados foram georreferenciados, e foi ponderada a densidade de sintomas por área. Nas 451 pessoas estudadas, o teste de Chi-Quadrado foi significativo para todos os sintomas, exceto chiado comparando gradientes de distância. Os sintomas agregados apresentaram as seguintes ODDS Ratio (OR): respiratórios altos (OR: 6,18; 2,10; 1,00); irritativos (OR: 6,06; 2,09; 1,00); respiratórios baixos (OR: 4,93; 2,08; 1,00); náuseas e dor de cabeça (OR: 4,66; 2,02; 1,00). Os autores encontraram maior densidade de sintomas nas proximidades da fonte emissora. O estudo contribuiu para desencadear medidas mitigadoras na empresa.
\end{abstract}

Palavras-chave: ácidos sulfúricos; acidentes químicos; vigilância.

\begin{abstract}
Following an environmental accident in a sulfuric acid production plant, a transversal study was made to investigate respiratory and irritative symptoms on the population. A questionnaire was applied to a simple random sample from people present until 900 meters from the plant by the time of the event, considering the winds' directions. The prevalence of symptoms and Chi-square test was made according to gradients of 300 meters. The density of symptoms per area, geo-referenced, was pondered. The Chi-square test was significant on the 451 people studied for all symptoms, except by wheezing, comparing gradients of distance. The grouped symptoms presented the following ODDS Ratio (OR): high respiratory (OR: 6.18; 2.10; 1.00); irritative (OR: 6.06; 2.09; 1.00); low respiratory (OR: 4.93; 2.08; 1.00); nausea and headache (OR: 4.66; 2.02; 1.00). The authors have found a major density of symptoms in the vicinities of the source. The study contributed to instigate mitigating measures in the company.
\end{abstract}

Keywords: sulfuric acid; chemical accident; surveillance.

\footnotetext{
Trabalho realizado pela Gerência de Vigilância em Saúde Ambiental (GVISAM) da Coordenação de Vigilância em Saúde (COVISA) da Secretaria Municipal de Saúde de São Paulo - São Paulo (SP), Brasil.

${ }^{1}$ Gerência de Vigilância em Saúde Ambiental da Coordenação de Vigilância em Saúde (GVISAM), Secretaria Municipal de Saúde (SMS) de São Paulo - São Paulo (SP), Brasil.

${ }^{2}$ Núcleo de Informação em Saúde da Coordenação de Vigilância em Saúde (COVISA), SMS - São Paulo (SP), Brasil.

${ }^{3}$ Supervisão de Vigilância em Saúde, SMS - São Paulo (SP), Brasil.

Endereço para correspondência: Clarice Umbelino de Freitas - Rua Santa Isabel, 188, $5^{\circ}$ andar, Vila Buarque - CEP: 01221-010 - São Paulo (SP), Brasil;

E-mail: umbelino.freitas@gmail.com

Fonte de financiamento: Secretaria Municipal de Saúde de São Paulo - São Paulo (SP), Brasil.

Conflito de interesse: nada a declarar.
} 


\section{INTRODUÇÃO}

Este artigo refere-se a acidente ocorrido por volta das $12 \mathrm{~h} 30$ na região central de um distrito do Município de São Paulo, Brasil. Pessoas que lá se encontravam foram surpreendidas por um forte odor e nuvem de fumaça, provenientes da retomada do processo de produção de uma grande indústria local, os quais, a partir do centro do distrito, se dispersavam na direção sul e sudeste. Houve pânico entre os transeuntes, que invadiam a estação de trem e as lojas. No atendimento da emergência, foi contabilizado o encaminhamento de 23 pessoas para atendimento médico por causa de problemas respiratórios agudos. Ao perceber que a direção dos ventos seguia para o centro do distrito, a indústria interrompeu a retomada do processo de produção. Pouco depois do acidente, ocorreu uma grande precipitação de chuva na região. No dia seguinte à ocorrência, técnicos da Vigilância da Municipalidade (COVISA) da Secretaria Municipal de Saúde de São Paulo compareceram ao local para proceder à investigação. A indústria em questão produz ácido sulfúrico em uma das plantas existentes em seu parque.

No processo de produção do ácido sulfúrico, realizado a partir do enxofre, o controle da temperatura é fundamental. Nas paradas de produção e posterior retomada do processo, quando a temperatura não atingiu seu ponto ideal, a conversão dessa matéria-prima em ácido sulfúrico é baixa, ocorrendo maiores emissões de poluentes $\left(\mathrm{SO}_{2} \mathrm{e} \mathrm{SO}_{3}\right)$ para a atmosfera ${ }^{1}$.

$\mathrm{O}$ dióxido de enxofre é um gás incolor e de odor forte e irritante cujo limite de percepção é de $3 \mathrm{ppm}^{2}$. Na atmosfera, é rapidamente oxidado em reações complexas envolvendo radicais livres de oxigênio, nitrogênio e hidrocarbonetos, formando trióxido de enxofre e principalmente ácido sulfúrico. $O$ trióxido de enxofre é também um gás incolor e quando em contato com o ar e a água nele existente apresenta-se como fumaça branca. Permanece no ambiente por pequenos períodos, pois reage com a água, transformando-se em ácido sulfúrico. $\mathrm{O}$ ácido sulfúrico é incolor e altamente corrosivo, com odor forte, e seu limite de percepção de $1 \mathrm{mg} / \mathrm{m}^{3}$ de ar. O dióxido de enxofre e o ácido sulfúrico são mais facilmente encontrados na atmosfera que o trióxido de enxofre. $\mathrm{O}$ ácido sulfúrico, em presença da água, pode formar gotículas que ficam suspensas na atmosfera por longos períodos, sendo removidas do ar com a chuva, o que contribui para a formação de chuva ácida ${ }^{3}$.

O ácido sulfúrico é corrosivo e irritante, provocando efeitos locais na pele, trato respiratório e gastrointestinal por contato direto. A respiração de gotículas de ácido sulfúrico provoca dificuldade para respirar e irritação do trato respiratório. Esses efeitos são mais pronunciados após exercício ou em indivíduos asmáticos. A inalação de gotículas de ácido sulfúrico pode ainda diminuir a capacidade do trato respiratório na remoção de outras partículas presentes no ar e que normalmente são expelidas. Ingestão de ácido sulfúrico causa irritação da boca e garganta, podendo levar à erosão do estômago. Em contato com os olhos ou pele, provoca queimadura química com irritação e vermelhidão locais, chegando a ferimento ou cegueira, dependendo das concentrações.

Entre os diversos segmentos da população, as crianças são mais sensíveis aos efeitos do ácido sulfúrico em razão do menor diâmetro do seu trato respiratório e por respirarem mais ar por quilo de peso que os adultos ${ }^{2}$.

O dióxido de enxofre é classificado como um gás irritante. A inalação aguda provoca broncoespasmo com sensação de sufocação, tosse, sibilos, dor no peito, cianose, náuseas e vômitos. Sobreviventes de exposições a altas concentrações de $\mathrm{SO}_{2}$ podem desenvolver doença obstrutiva ou restritiva do pulmão e hiper-reatividade brônquica. Os asmáticos são particularmente sensíveis aos efeitos do dióxido de enxofre. Em decorrência da presença de água nos diversos órgãos e aparelhos, os efeitos da exposição a trióxido de enxofre são similares aos do ácido sulfúrico 4 .

Em exposições correntes a $\mathrm{SO}_{2}$, são descritos aumento de hospitalizações por asma e por doença pulmonar obstrutiva crônica, aumento da mortalidade geral e outros efeitos relacionados ao pulmão, como hiper-reatividade brônquica e diminuição da função pulmonar ${ }^{4}$. Por causa de seus riscos à saúde, a Agência de Proteção Ambiental Americana (EPA) estabelece, como padrão de qualidade do ar, concentrações de $\mathrm{SO}_{2}$ de 0.03 ppm (média aritmética anual); 0.14 ppm média de 24 horas e $0.5 \mathrm{ppm}\left(1300 \mu \mathrm{g} / \mathrm{m}^{3}\right)$ como média de 3 horas. Os dois últimos valores não podem ser excedidos mais de uma vez ao ano. A legislação brasileira segue os padrões estabelecidos pela EPA.

Este episódio levou o setor de Vigilância do município a realizar estudo que teve como objetivo avaliar os efeitos de curto prazo na saúde dos moradores e trabalhadores locais, com vistas a estimar o impacto dessa retomada do processo de produção e subsidiar a adoção de medidas de prevenção de novas ocorrências dessa natureza.

\section{MÉTODOS}

Foi realizado estudo transversal em inquérito recordatório, referente ao dia da ocorrência do acidente, de sintomas respiratórios, irritativos e de percepção de odor numa amostra das pessoas residentes ou trabalhadoras da área de influência da empresa. Para escolha dos sintomas a serem investigados, se tomou como base a descrição da literatura de efeitos decorrentes de exposição aguda a $\mathrm{SO}_{2}, \mathrm{SO}_{3}$ e ácido sulfúrico. Tendo 
como base os dados de direção e velocidade dos ventos no momento da ocorrência, foi delimitada área de influência, com distâncias estimadas de 300 até $900 \mathrm{~m}$ a sul, sudoeste e sudeste, a partir da empresa ferroviária local.

A população residente foi estimada utilizando-se os dados dos setores censitários de 2000, e foi calculada amostra aleatória simples considerando-se uma prevalência de sintomas de $10 \%$ e erro de $5 \%$ para cada extrato de $300 \mathrm{~m}$ (Tabela 1) em que se estimou 212 residentes no primeiro extrato, 962 no segundo e 3.746 no terceiro. A área de estudo inclui o centro do distrito onde seus imóveis são predominantemente comerciais. A partir do registro de imóveis do Serviço de Controle de Zoonoses, foi sorteado o primeiro a ser visitado e o intervalo amostral com base na população. Os imóveis, residenciais ou comerciais, foram visitados até completar toda a área, considerando-se o intervalo amostral, tendo-se ultrapassado a amostra inicialmente proposta, em todos os extratos de $300 \mathrm{~m}$.

Foram entrevistados apenas adultos e estabeleceu-se como critério de inclusão na pesquisa estar no local (residência ou comércio) no dia da ocorrência do acidente. $\mathrm{Na}$ primeira questão ao entrevistado, perguntava-se se estava no local no dia da ocorrência. Caso sim, dava-se continuidade à entrevista; caso contrário, se inquiria sobre a presença de outra pessoa do imóvel, presente no local neste dia. Quando não se encontrava residente ou comerciante no imóvel neste dia, prosseguia-se para o novo imóvel da amostra. Em cada imóvel, foi entrevistado um morador ou responsável pelo estabelecimento.

As questões do inquérito se referiam a identificação, endereço, presença de sintomas respiratórios, irritativos, percepção de odor e possível procura por serviço médico em decorrência do acidente, além de observações livres para o entrevistado. Foram estabelecidos como critérios de exclusão não lembrar ou não se encontrar no imóvel no dia da ocorrência do acidente.

Os dados foram colhidos pelos agentes de controle de zoonoses, iniciando-se cinco dias após a ocorrência do evento. A equipe realizou todas as entrevistas no período de um mês, iniciando o trabalho pelas residências mais próximas da fonte. $\mathrm{Na}$ área $1(300 \mathrm{~m})$, foi excluído um participante por não ter assinado o termo de consentimento apesar de ter concordado em responder ao questionário. Particularmente na área 3 (900 m), alguns entrevistados não lembravam se estavam em casa no dia da ocorrência do acidente ou não tinham notícia do ocorrido. Os que não lembravam se estavam no local neste dia foram excluídos das análises.

Cada pessoa entrevistada foi esclarecida sobre a finalidade do inquérito e solicitada a fornecer seu consentimento por
Tabela 1. Dados de população residente e imóveis da área do entorno da empresa (900 m) e proposta amostral - São Paulo (SP), 2006

\begin{tabular}{lcccr} 
Descrição & $\mathbf{3 0 0} \mathbf{~ m}$ & $\mathbf{6 0 0 ~ m}$ & $\mathbf{9 0 0 ~} \mathbf{m}$ & Total \\
População residente estimada $^{(\mathrm{a})}$ & 212 & 962 & 3746 & 4920 \\
Imóveis(b) $^{(b)}$ & 519 & 444 & 1337 & 2300 \\
Amostra proposta & 84 & 122 & 134 & 340 \\
Entrevistas realizadas & 136 & 129 & 224 & 489 \\
Entrevistas elegíveis & 135 & 123 & 193 & 451 \\
\hline
\end{tabular}

(a)População residente estimada do censo de 2000

(b)Número de imóveis residenciais ou comerciais do cadastro do Serviço de Controle de Zoonoses

escrito. Garantiu-se a todos o sigilo quanto à identificação do nome e endereço. O presente estudo foi aprovado pelo Comitê de Ética do Instituto Adolfo Lutz e financiado com recursos da municipalidade de São Paulo.

\section{Análise estatística}

Foi calculada a prevalência de cada um dos sintomas e de sua agregação segundo distância de cada $300 \mathrm{~m}$ da fonte, e realizado o teste de $\chi^{2}$. A amostra foi agrupada segundo presença ou ausência de sintomas e distância da fonte e foi efetuado teste de $\chi^{2}$ de tendência linear para cada queixa e queixas agrupadas, tomando-se como baseline a distância de $900 \mathrm{~m}$. O agrupamento tanto para o cálculo da prevalência quanto para o teste de tendência linear obedeceu aos seguintes critérios:

(1) Todos os sintomas respiratórios: dificuldade de respirar, irritação na garganta, tosse, irritação no nariz, chiado no peito.

(2) Sintomas irritativos: irritação na garganta, tosse, irritação no nariz, irritação nos olhos.

(3) Respiratórios baixos: dificuldade de respirar, chiado no peito.

(4) Outros: dor de cabeça, náuseas.

A percepção de odor foi analisada separadamente. Foi estabelecida como significativa uma probabilidade menor que $5 \%(\alpha<0,05)$.

A amostra foi georreferenciada segundo o endereço de residência do entrevistado, construindo-se um mapa de pontos com a tabela de sintomas agregados. Utilizou-se o estimador de densidade de Kernel para estabelecer a concentração espacial dos sintomas relatados ${ }^{5,6}$. Tal estimativa foi calculada a partir da distância média entre os casos, utilizando a função quártica de alisamento e o raio adaptativo como parâmetro de proximidade. Posteriormente, foram criados buffers de proximidade entre as áreas densas em sintomas e as diferentes distâncias em relação à indústria. $\mathrm{Na}$ análise dos dados, foram utilizados os aplicativos SPSS 16, Maptitude e Crimestat ${ }^{7}$. 
Tabela 2. Prevalência de sintomas e percepção de odores segundo faixas de distância da empresa - São Paulo (SP), 2006

\begin{tabular}{|c|c|c|c|c|c|c|c|}
\hline \multirow{3}{*}{ Sintomas } & \multicolumn{5}{|c|}{ Área } & & \multirow{3}{*}{ Valor $\mathrm{p}$} \\
\hline & \multicolumn{2}{|l|}{$300 \mathrm{~m}$} & \multicolumn{2}{|l|}{$600 \mathrm{~m}$} & \multicolumn{2}{|l|}{$900 \mathrm{~m}$} & \\
\hline & Sim & Não & Sim & Não & Sim & Não & \\
\hline Dificuldade de respirar & $54(40 \%)$ & 81 & $26(21 \%)$ & 97 & $22(11 \%)$ & 171 & 0.00 \\
\hline Ardor na garganta & $47(35 \%)$ & 88 & $24(20 \%)$ & 99 & $24(12 \%)$ & 169 & 0.00 \\
\hline Tosse & $50(37 \%)$ & 85 & $27(22 \%)$ & 96 & $19(10 \%)$ & 174 & 0.00 \\
\hline Coceira no nariz & $45(33 \%)$ & 90 & $21(17 \%)$ & 102 & $22(11 \%)$ & 171 & 0.00 \\
\hline Ardor nos olhos & $37(27 \%)$ & 98 & $19(15 \%)$ & 104 & $19(10 \%)$ & 174 & 0.00 \\
\hline Chiado no peito & $11(8 \%)$ & 124 & $8(6,5 \%)$ & 115 & $5(3 \%)$ & 188 & 0.06 \\
\hline Dor de cabeça & $31(23 \%)$ & 104 & $15(12 \%)$ & 108 & $14(7 \%)$ & 179 & 0.00 \\
\hline Náuseas & $23(17 \%)$ & 112 & $11(9 \%)$ & 112 & $7(4 \%)$ & 186 & 0.00 \\
\hline Percepção de odor & $99(73 \%)$ & 36 & $49(40 \%)$ & 74 & $47(24 \%)$ & 146 & 0.00 \\
\hline
\end{tabular}

Tabela 3. ODDS Ratio e Intervalo de Confiança de 95\% para sintomas e percepção do odor referidos pela população, segundo gradientes de distância da empresa - São Paulo (SP), 2006

\begin{tabular}{lccc} 
Queixas referidas & & OR & IC95\% \\
Respiratórias & $300 \mathrm{~m}$ & 6,18 & $3,74-10,19$ \\
& $600 \mathrm{~m}$ & 2,10 & $1,24-3,55$ \\
& $900 \mathrm{~m}$ & 1,00 & baseline \\
\hline \multirow{3}{*}{ Irritativas } & $300 \mathrm{~m}$ & 6,06 & $3,65-10,05$ \\
& $600 \mathrm{~m}$ & 2,09 & $1,22-3,57$ \\
& $900 \mathrm{~m}$ & 1,00 & baseline \\
\hline \multirow{3}{*}{ Respiratórias baixas } & $300 \mathrm{~m}$ & 4,93 & $2,83-8,58$ \\
& $600 \mathrm{~m}$ & 2,08 & $1,13-3,82$ \\
& $900 \mathrm{~m}$ & 1,00 & baseline \\
\hline \multirow{3}{*}{ Outras (náuseas e cefaleia) } & $300 \mathrm{~m}$ & 4,66 & $2,48-8,75$ \\
& $600 \mathrm{~m}$ & 2,02 & $1,00-4,10$ \\
& $900 \mathrm{~m}$ & 1,00 & baseline \\
\hline \multirow{3}{*}{ Percepção de odor } & $300 \mathrm{~m}$ & 8,54 & $5,16-14,13$ \\
& $600 \mathrm{~m}$ & 2,06 & $1,26-3,35$ \\
& $900 \mathrm{~m}$ & 1,00 & baseline \\
\hline
\end{tabular}

\section{RESULTADOS}

A Tabela 1 apresenta os dados populacionais, de estabelecimentos, amostra projetada e realizada, bem como o número de entrevistas elegíveis.

Foram analisados os dados de 451 pessoas, sendo 293 (65\%) trabalhadores em estabelecimentos comerciais e 158 (35\%) moradores. A média de idade do grupo entrevistado foi de 39 anos (desvio-padrão: 16), com 259 mulheres (57\%) e 192 homens (43\%). Da amostra, sete pessoas relataram ter buscado algum tipo de cuidado à saúde na questão aberta.

A Tabela 2 apresenta a prevalência de sintomas e percepção do odor segundo faixas de distância da fonte, na qual pode ser visto que houve diferença significativa entre elas para os sintomas em separado, exceto para chiado no peito quando considerado isoladamente. $\mathrm{O}$ teste de tendência linear foi significativo para todos os sintomas considerados isoladamente.

A Tabela 3 apresenta o teste de tendência linear segundo distância para os sintomas agregados e a percepção do odor.
Em todos os casos, o valor $\mathrm{p}$ foi menor que 0,05 , mostrando que quanto mais próxima da fonte de emissão dos poluentes mais intensa foi a exposição, e observou maior risco de ocorrência de sintomas e de percepção do odor.

A análise dos dados georreferenciados dos sintomas respiratórios e irritativos é apresentada na Figura 1, tendo-se maior densidade na área próxima à empresa emissora. É importante relatar que, quando georreferenciados, tanto sintomas quanto percepção do odor tiveram comportamento similar.

\section{DISCUSSÃO}

Foi encontrada neste estudo maior prevalência de sintomas respiratórios, irritativos e de percepção do odor na área mais próxima da empresa, no dia da ocorrência do acidente. O risco de apresentar sintomas diminui à medida que a população está mais distante da fonte. Esses achados indicam que o episódio impactou a saúde da população do entorno da empresa.

Os sintomas investigados neste inquérito são em geral leves e de curto prazo. Os estudos referidos na literatura partem de sintomatologia de maior gravidade e abrangem maior período ${ }^{8,9}$. No momento do acidente, não se dispunha de informações de prevalência de sintomas no ponto, ou seja, para um dia. O presente estudo baseou-se em uma possível prevalência de $10 \%$ utilizada para o cálculo da amostra em Pirapora do Bom Jesus, em recordatório de 15 dias, para comparação com o município de São Lourenço da Serra, livre do poluente em estudo $\left(\mathrm{H}_{2} \mathrm{~S}\right)^{10}$. Vale comentar que a amostra foi calculada para populações independentes, garantindo-se a aleatoriedade no sorteio dos participantes. Apesar de a amostra proposta partir de uma prevalência para um recordatório de 15 dias e ter sido calculada para populações independentes, estando, portanto, aparentemente subestimada, foi capaz de detectar diferença significativa entre os grupos. 

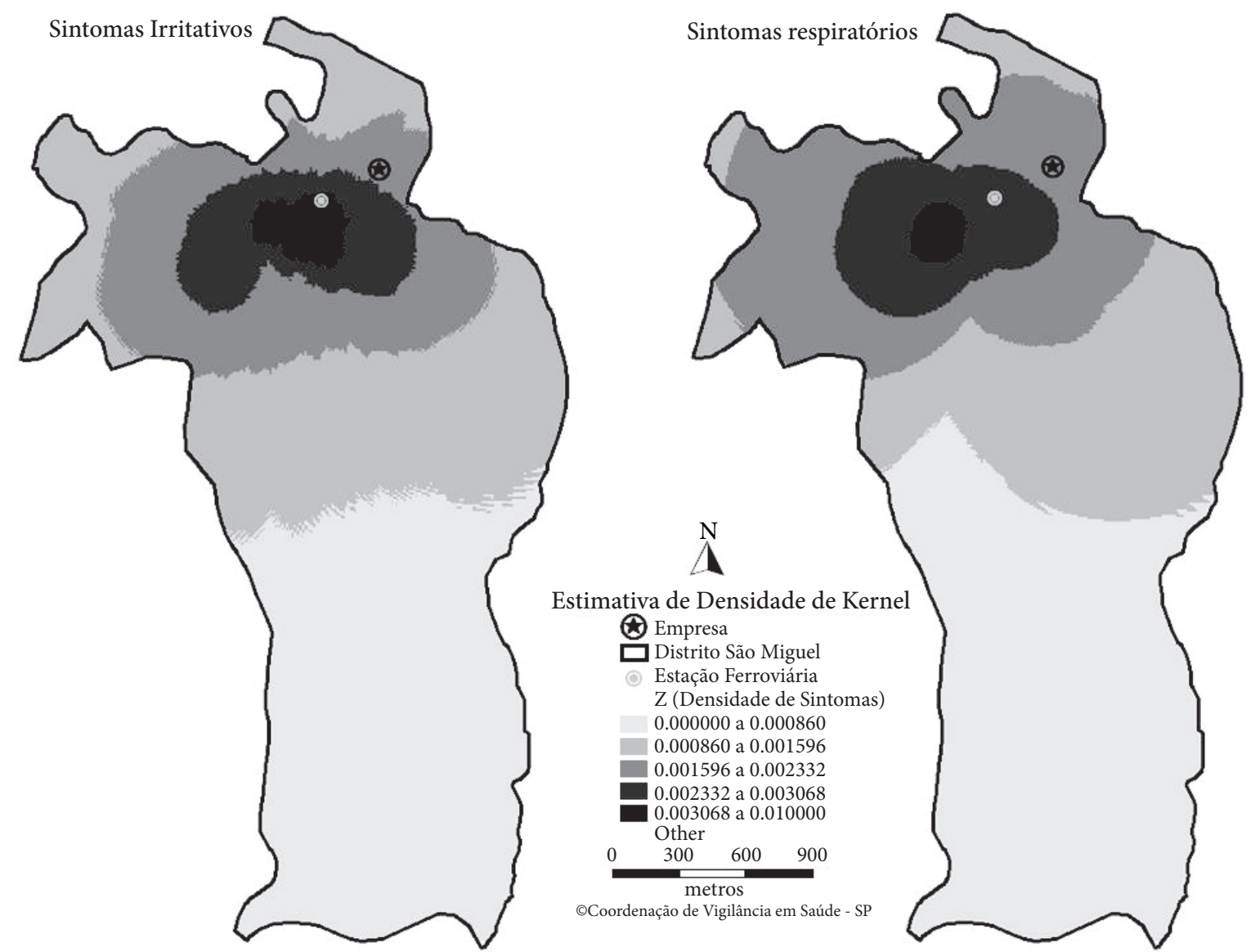

Figura 1. Densidade de sintomas respiratórios e irritativos referidos no dia do acidente ambiental - São Paulo (SP), 2006.

Buscou-se realizar a investigação no menor intervalo de tempo possível. Em estudos de recordatório, deve-se levar em conta o viés de memória. O presente inquérito teve a duração de um mês, e provavelmente a população da faixa de distância de 900 m apresentou a prevalência de sintomas próxima do esperado para exposições correntes de contaminação do ar, como as encontradas em São Paulo. Esse grupo foi usado como baseline para comparação com os demais, mais próximos da fonte emissora.

Episódios agudos de poluição do ar envolvendo o $\mathrm{SO}_{2}$ são citados na literatura, particularmente anteriores a todo movimento internacional regulamentador decorrente da dramaticidade dessas ocorrências ${ }^{11}$.

O primeiro episódio documentado ocorreu em 1930, no Vale do Meuse, Bélgica, quando foram descritos aumento de doenças respiratórias, complicações de doenças cardiovasculares e excesso de 60 mortes até dois dias após o episódio de nevoeiro que cobriu a cidade ${ }^{12}$.

Em 1948, entre os dias 26 e 31 de outubro, outra tragédia em Donora, na Pensilvânia, causou excesso de 17 mortes. A cidade que foi palco da ocorrência contava com apenas 14.000 habitantes, dos quais $43 \%$ foram afetados ${ }^{11}$. Não dispomos dos níveis de contaminantes do ar nesses episódios. No ano de 1961, foi publicado estudo de acompanhamento de dez anos da população de Donora, tendo-se concluído por maiores prevalências de doenças e mortalidade entre os sobreviventes que, na época do acidente, apresentaram sintomatologia grave ${ }^{13}$.

Estudo realizado por Logan $(1953)^{14}$, em Londres, relativo ao episódio de poluição aguda ocorrido de 5 a 9 de dezembro de 1952, estima a ocorrência de 4.000 mortes. Nesse período, os níveis de $\mathrm{SO}_{2}$ alcançaram a média de $1,34 \mathrm{ppm}^{15}$. A estimativa de $\mathrm{SO}_{2}$ efetuada para a área do presente estudo no raio de $500 \mathrm{~m}$, a partir do modelo de dispersão gaussiano de Pasquill-Gifford, encontrou concentração máxima de 9,5 ppm no dia da ocorrência do acidente, considerando um cenário conservador $^{16}$, e de 5,5 ppm na madrugada do dia seguinte. Nesta madrugada, a medida de aferição do modelo realizada no ponto foi de 5,4 $\mathrm{ppm}^{17}$. No episódio londrino, os níveis de fumaça apresentaram concentração máxima de $4,46 \mathrm{mg} / \mathrm{m}^{3}$.

Mais recentemente (1998), na cidade de Nottinghan, Inglaterra, ocorreu episódio de poluição por $\mathrm{SO}_{2}$. $\mathrm{Na}$ semana dessa ocorrência, detectou-se excesso de 25 internações hospitalares quando comparadas com as semanas anteriores ${ }^{18}$. 
Não foram encontrados estudos de sintomas leves, de curto prazo, decorrentes de exposição aguda a $\mathrm{SO}_{2}$ que pudessem ser utilizados para comparação com o presente inquérito.

Considerando os estudos citados de episódios agudos de poluição e os níveis de $\mathrm{SO}_{2}$ estimados no dia da ocorrência do acidente analisado no presente trabalho, podemos supor que estaríamos diante de uma tragédia de grandes proporções caso as condições de dispersão dos ventos não fossem favoráveis e a retomada do processo de produção não fosse interrompida.

\section{CONCLUSÕES}

Em se tratando de estudo da área de Vigilância, este trabalho teve como objetivo final dar subsídios às ações de controle sanitário. Para isso, foi encaminhado relatório das análises para a Vigilância em Saúde do Trabalhador da Coordenação de Vigilância em Saúde (COVISA/SP) e para o órgão ambiental do estado (CETESB), solicitando que providências fossem tomadas para prevenir a recorrência de acidentes dessa natureza.

Com base na análise do processo de produção da empresa, foram feitas várias exigências legais com vistas à prevenção de acidentes ambientais. As informações da CETESB dão conta do cumprimento das exigências, com a modernização e readequação do processo de produção da planta de produção de ácido sulfúrico.
O diagnóstico do impacto na saúde da população decorrente de exposições ambientais adversas ou após acidentes maiores pode ser uma estratégia para as atividades de vigilância, contribuindo para a atuação dos órgãos de controle, tanto do Setor Ambiental quanto do Setor Saúde. Enquanto estratégia, não deve perder de vista o princípio da precaução. Em outras palavras, não se faz necessário esperar as vítimas. No entanto, se as vítimas existem, devem ser mostradas exaustivamente, exigindo a melhoria do ambiente e prevenção de novos danos à saúde das pessoas. Em nosso meio, outras investigações, envolvendo os órgãos afetos (Saúde e Ambiente) e o Ministério Público, já serviram de subsídio para o estabelecimento de medidas preventivas de danos, de remediação ou de ressarcimento, constituindo-se em atividades de promoção da saúde $e^{10,19}$.

\section{CONTRIBUIÇÃO DOS AUTORES}

Clarice Umbelino de Freitas participou da concepção e elaboração do projeto, análise dos dados e elaboração do texto final. Pedro José Villaça realizou as análises dos dados georreferenciados e participou da elaboração do texto final. Carolina Beltramini de Carvalho Donola, Débora Moraes Coelho, Ana Claudia Furlan Mori e Marlene Tursi Zampietro participaram das análises e revisão do texto final. André Pereira Leite participou da concepção e desenho do estudo.

\section{REFERÊNCIAS}

1. MB Consultores Ltda. Processo de produção do ácido sulfúrico. [cited 2011 Ago 23]. Available from: http://www.h2so4.com.br/h2so4/ download/arquivos/manualdeacidosulfurico\%20.pdf

2. Agency for Toxic Substances and Disease Registry (ATSDR). 1998. Toxicological profile for Sulfur Dioxide. Atlanta, GA: U.S. Department of Health and Human Services; 1998.

3. Agency for Toxic Substances and Disease Registry (ATSDR). 1998. Toxicological profile for Sulfur Trioxide and Sulfuric Acid. Atlanta, GA: U.S. Department of Health and Human Services; 1998.

4. MEDITEXT. Sulphur dioxide. In: Meditextâ Medical Management [database on CR-ROM]. 2010. Greewood Village, Colo: Thompson Micromedex; 2010.

5. Bailey TC, Gatrell AC. Interactive Spatial Data Analysis. New York: John Willey and Sons; 1995.

6. Cromley EK, Mclafferty SL. GIS and Public Health. New York: The Guilford Press; 2002.

7. Levine N. Crimestat 2 [manual]. National Institute of Justice. 2000.

8. Carandina L. Prevalência de sintomas sugestivos de doenças respiratórias inespecíficas na população urbana de Botucatu, São Paulo. [tese] São
Paulo: Faculdade de Saúde Pública da Universidade de São Paulo, Departamento de Epidemiologia; 1986.

9. Ribeiro H, Cardoso MR. Air pollution and children's health in São Paulo (1986-1998). Social Sci Med. 2003:57(11);2013-22.

10. Freitas CU, Grimaldi R, Ushirobira MFH, Oliveira MC, Martins MH, Sano L, et al. Prevalence of respiratory symptoms in Pirapora do Bom Jesus exposed to sulfur reduced composts compared with control area. São Paulo, Brazil, 2004. Epidemiology. 2006:17(Supl 6). Abstracts P-017.

11. Bascon R, Brombreg PA, Costa DA, Devlin R, Dockery DW, Framptom MW, et al. Health effects of outdoor air pollution. Committee of Environmental and Occupational Health Assembly of the American Thoracic Society. Am J Respir Crit Care Med. 1996;153(1):3-50.

12. Firket M. Sur les causes des accidents suvenus dans la Vallée de la Meuse, lors des brouillards de décembre 1930. Bull Acad Roy Med Belg. 1931;11:683-741.

13. Ciocco A, Thompson, DJ. A follow-up of Donora ten years after: methodology and findings. Am J Public Health Nations Health.1961;51(2):155-164.

14. Logan WPD. Mortality in the London fog incident, 1952. Lancet. 1953:1(6755):336-8. 
15. Ministry of Health. Mortality and morbidity during the London fog of December 1952. London: HMSO; 1954 (Reports on Public Health and Medical Subjects, nº 95 1954).

16. Freitas CU, Martins-Jr L, Donola CBC, Mori AC, Vilaça PJ, Leite AP. Multiple approaches for an environmental accident on a sulfuric acid plant. Salud Publica de Mexico. 2007;49(ed especial 2). Abstract 308. P ES09.

17. Martins-Junior L, Lorenzi RL. Acidente químico com dióxido de enxofre em um populoso distrito de uma grande metrópole: cenários de exposição a partir de um modelo Gaussiano de dispersão. Rev Bras Saúde Ocup. 2007;32(116):31-7.
18. Brown TP, Rushton L, Mugglestone MA, Meechan DF. Health effects of a sulphur dioxide air pollution episode. J Public Health Med. 2003;25(4):369-71.

19. Freitas CU, De Capitani EM, Gouveia N, Simonetti MH, Paula e Silva MR, Kira CS et al. Lead exposure in an urban community: investigation of risk factors and assessment of the impact of lead abatement measures. Environ Res. 2007;103:338-44.

Recebido em: 10/05/2012 Aprovado em: 10/07/2012 